\title{
Sobolev space, Besov space and Triebel-Lizorkin space on the Laguerre hypergroup
}

\section{Jizheng Huang*}

"Correspondence:

hjzheng@163.com

College of Sciences, North China

University of Technology, Beijing,

100144, China

\begin{abstract}
In this paper, we will investigate function spaces, including a Sobolev space, a Besov space and a Triebel-Lizorkin space, on the Laguerre hypergroup.

MSC: 42B20; 42B25; 42C05
\end{abstract}

Keywords: Laguerre hypergroup; Sobolev space; Besov space; Triebel-Lizorkin space

\section{Introduction and preliminaries}

In [1] and [2], the authors investigated a Sobolev space on the dual of the Laguerre hypergroup and a generalized Besov space on the Laguerre hypergroup. In this paper, we define a Sobolev space on the Laguerre hypergroup by the Bessel potential. Then, we define a Besov space by the real interpolation of a Sobolev space and prove that our definition is a generalization of that given in [1]. For the completeness, we also study a Triebel-Lizorkin space on the Laguerre hypergroup.

We first give some notations about the Laguerre hypergroup. Let $\mathbf{K}=[0, \infty) \times \mathbf{R}$ equipped with the measure

$$
d m_{\alpha}(x, t)=\frac{1}{\pi \Gamma(\alpha+1)} x^{2 \alpha+1} d x d t, \quad \alpha \geq 0 .
$$

We denote by $L_{\alpha}^{p}(\mathbf{K})$ the spaces of measurable functions on $\mathbf{K}$ such that $\|f\|_{\alpha, p}<+\infty$, where

$$
\begin{aligned}
\|f\|_{\alpha, p} & =\left(\int_{\mathbf{K}}|f(x, t)|^{p} d m_{\alpha}(x, t)\right)^{\frac{1}{p}}, \quad 1 \leq p<\infty \\
\|f\|_{\alpha, \infty} & =\operatorname{esssup}_{(x, t) \in \mathbf{K}}|f(x, t)| .
\end{aligned}
$$

For $(x, t) \in \mathbf{K}$, the generalized translation operators $T_{(x, t)}^{(\alpha)}$ are defined by

$$
\begin{aligned}
& T_{(x, t)}^{(\alpha)} f(y, s) \\
& \quad=\left\{\begin{array}{l}
\frac{1}{2 \pi} \int_{0}^{2 \pi} f\left(\sqrt{x^{2}+y^{2}+2 x y \cos \theta}, s+t+x y \sin \theta\right) d \theta, \quad \text { if } \alpha=0, \\
\frac{\alpha}{\pi} \int_{0}^{2 \pi} \int_{0}^{1} f\left(\sqrt{x^{2}+y^{2}+2 x y r \cos \theta}, s+t+x y r \sin \theta\right) r\left(1-r^{2}\right)^{\alpha-1} d r d \theta, \quad \text { if } \alpha>0 .
\end{array}\right.
\end{aligned}
$$

(c) 2012 Huang; licensee Springer. This is an Open Access article distributed under the terms of the Creative Commons Attribution License (http://creativecommons.org/licenses/by/2.0), which permits unrestricted use, distribution, and reproduction in any medium, provided the original work is properly cited. 
It is known that $T_{(x, t)}^{(\alpha)}$ satisfies

$$
\left\|T_{(x, t)}^{(\alpha)} f\right\|_{\alpha, p} \leq\|f\|_{\alpha, p}
$$

Let $M_{b}(\mathbf{K})$ denote the space of bounded Radon measures on $\mathbf{K}$. The convolution on $M_{b}(\mathbf{K})$ is defined by

$$
(\mu * v)(f)=\int_{\mathbf{K} \times \mathbf{K}} T_{(x, t)}^{(\alpha)} f(y, s) d \mu(x, t) d \nu(y, s) .
$$

It is easy to see that $\mu * v=v * \mu$. If $f, g \in L_{\alpha}^{1}(\mathbf{K})$ and $\mu=f m_{\alpha}, v=g m_{\alpha}$, then $\mu * v=(f * g) m_{\alpha}$, where $f * g$ is the convolution of functions $f$ and $g$ defined by

$$
(f * g)(x, t)=\int_{\mathbf{K}} T_{(x, t)}^{(\alpha)} f(y, s) g(y,-s) d m_{\alpha}(y, s) .
$$

The following lemma follows from (1).

Lemma 1 Let $f \in L_{\alpha}^{1}(\mathbf{K})$ and $g \in L_{\alpha}^{p}(\mathbf{K}), 1 \leq p \leq \infty$. Then

$$
\|f * g\|_{\alpha, p} \leq\|f\|_{\alpha, 1}\|g\|_{\alpha, p} .
$$

$(\mathbf{K}, *, i)$ is a hypergroup in the sense of Jewett $(c f .[4,9])$, where $i$ denotes the involution defined by $i(x, t)=(x,-t)$. If $\alpha=n-1$ is a nonnegative integer, then the Laguerre hypergroup $\mathbf{K}$ can be identified with the hypergroup of radial functions on the Heisenberg group $\mathbb{H}^{n}$.

The dilations on $\mathbf{K}$ are defined by

$$
\delta_{r}(x, t)=\left(r x, r^{2} t\right), \quad r>0 .
$$

It is clear that the dilations are consistent with the structure of the hypergroup. Let

$$
f_{r}(x, t)=r^{-(2 \alpha+4)} f\left(\frac{x}{r}, \frac{t}{r^{2}}\right) .
$$

Then we have

$$
\left\|f_{r}\right\|_{\alpha, 1}=\|f\|_{\alpha, 1}
$$

We also introduce a homogeneous norm defined by $|(x, t)|=\left(x^{4}+4 t^{2}\right)^{\frac{1}{4}}(c f$. [11]). Then we can define the ball centered at $(0,0)$ of radius $r$, i.e., the set $B_{r}=\{(x, t) \in \mathbf{K}:|(x, t)|<r\}$.

Let $f \in L_{\alpha}^{1}(\mathbf{K})$. Set $x=\rho(\cos \theta)^{\frac{1}{2}}, t=\frac{1}{2} \rho^{2} \sin \theta$. We get

$$
\begin{aligned}
& \int_{\mathbf{K}} f(x, t) d m_{\alpha}(x, t) \\
& \quad=\frac{1}{2 \pi \Gamma(\alpha+1)} \int_{-\frac{\pi}{2}}^{\frac{\pi}{2}} \int_{0}^{\infty} f\left(\rho(\cos \theta)^{\frac{1}{2}}, \frac{1}{2} \rho^{2} \sin \theta\right) \rho^{2 \alpha+3}(\cos \theta)^{\alpha} d \rho d \theta .
\end{aligned}
$$


If $f$ is radial, i.e., there is a function $\psi$ on $[0, \infty)$ such that $f(x, t)=\psi(|(x, t)|)$, then

$$
\begin{aligned}
\int_{\mathbf{K}} f(x, t) d m_{\alpha}(x, t) & =\frac{1}{2 \pi \Gamma(\alpha+1)} \int_{-\frac{\pi}{2}}^{\frac{\pi}{2}}(\cos \theta)^{\alpha} d \theta \int_{0}^{\infty} \psi(\rho) \rho^{2 \alpha+3} d \rho \\
& =\frac{\Gamma\left(\frac{\alpha+1}{2}\right)}{2 \sqrt{\pi} \Gamma(\alpha+1) \Gamma\left(\frac{\alpha}{2}+1\right)} \int_{0}^{\infty} \psi(\rho) \rho^{2 \alpha+3} d \rho .
\end{aligned}
$$

Specifically,

$$
m_{\alpha}\left(B_{r}\right)=\frac{\Gamma\left(\frac{\alpha+1}{2}\right)}{4 \sqrt{\pi}(\alpha+2) \Gamma(\alpha+1) \Gamma\left(\frac{\alpha}{2}+1\right)} r^{2 \alpha+4} .
$$

We consider the partial differential operator

$$
L=-\left(\frac{\partial^{2}}{\partial x^{2}}+\frac{2 \alpha+1}{x} \frac{\partial}{\partial x}+x^{2} \frac{\partial^{2}}{\partial t^{2}}\right)
$$

$L$ is positive and symmetric in $L_{\alpha}^{2}(\mathbf{K})$, and is homogeneous of degree 2 with respect to the dilations defined above. When $\alpha=n-1, L$ is the radial part of the sublaplacian on the Heisenberg group $\mathbb{H}^{n}$. We call $L$ the generalized sublaplacian.

Let $L_{m}^{(\alpha)}$ be the Laguerre polynomial of degree $m$ and order $\alpha$ defined in terms of the generating function by

$$
\sum_{m=0}^{\infty} s^{m} L_{m}^{(\alpha)}(x)=\frac{1}{(1-s)^{\alpha+1}} \exp \left(-\frac{x s}{1-s}\right)
$$

For $(\lambda, m) \in \mathbf{R} \times \mathbf{N}$, we put

$$
\varphi_{(\lambda, m)}(x, t)=\frac{m ! \Gamma(\alpha+1)}{\Gamma(m+\alpha+1)} e^{i \lambda t} e^{-\frac{1}{2}|\lambda| x^{2}} L_{m}^{(\alpha)}\left(|\lambda| x^{2}\right) .
$$

The following proposition summarizes some basic properties of functions $\varphi(\lambda, m)$.

Proposition 1 The function $\varphi_{(\lambda, m)}$ satisfies

(a) $\left\|\varphi_{(\lambda, m)}\right\|_{\alpha, \infty}=\varphi_{(\lambda, m)}(0,0)=1$,

(b) $\varphi_{(\lambda, m)}(x, t) \varphi_{(\lambda, m)}(y, s)=T_{(x, t)}^{(\alpha)} \varphi_{(\lambda, m)}(y, s)$,

(c) $L \varphi_{(\lambda, m)}=4|\lambda|\left(m+\frac{\alpha+1}{2}\right) \varphi_{(\lambda, m)}$.

Let $f \in L_{\alpha}^{1}(\mathbf{K})$, the generalized Fourier transform of $f$ is defined by

$$
\hat{f}(\lambda, m)=\int_{\mathbf{K}} f(x, t) \varphi_{(-\lambda, m)}(x, t) d m_{\alpha}(x, t) .
$$

It is easy to know that

$$
(f * g)^{\wedge}(\lambda, m)=\hat{f}(\lambda, m) \hat{g}(\lambda, m)
$$

and

$$
\hat{f}_{r}(\lambda, m)=\hat{f}\left(r^{2} \lambda, m\right) .
$$


Let $d \gamma_{\alpha}$ be the positive measure defined on $\mathbf{R} \times \mathbf{N}$ by

$$
\int_{\mathbf{R} \times \mathbf{N}} g(\lambda, m) d \gamma_{\alpha}(\lambda, m)=\sum_{m=0}^{\infty} \frac{\Gamma(m+\alpha+1)}{m ! \Gamma(\alpha+1)} \int_{\mathbf{R}} g(\lambda, m)|\lambda|^{\alpha+1} d \lambda .
$$

Write $L_{\alpha}^{p}(\hat{\mathbf{K}})$ instead of $L^{p}\left(\mathbf{R} \times \mathbf{N}, d \gamma_{\alpha}\right)$. We have the following Plancherel formula:

$$
\|f\|_{\alpha, 2}=\|\hat{f}\|_{L_{\alpha}^{2}(\hat{\mathbf{K}})}, \quad f \in L_{\alpha}^{1}(\mathbf{K}) \cap L_{\alpha}^{2}(\mathbf{K}) .
$$

Then the generalized Fourier transform can be extended to the tempered distributions. We also have the inverse formula of the generalized Fourier transform

$$
f(x, t)=\int_{\mathbf{R} \times \mathbf{N}} \hat{f}(\lambda, m) \varphi_{(\lambda, m)}(x, t) d \gamma_{\alpha}(\lambda, m)
$$

provided $\hat{f} \in L_{\alpha}^{1}(\hat{\mathbf{K}})$.

In the following, we give some basic properties about the heat kernel whose proofs can be found in [7]. Let $\left\{H^{s}\right\}=\left\{e^{-s L}\right\}$ be the heat semigroup generated by $L$. There is a unique smooth function $h((x, t), s)=h_{s}(x, t)$ on $\mathbf{K} \times(0,+\infty)$ such that

$$
H^{s} f(x, t)=f * h_{s}(x, t) .
$$

We call $h_{s}$ the heat kernel associated to $L$. We have

$$
h_{s}(x, t)=\int_{\mathbf{R}}\left(\frac{\lambda}{2 \sinh (2 \lambda s)}\right)^{\alpha+1} e^{-\frac{1}{2} \lambda \operatorname{coth}(2 \lambda s) x^{2}} e^{i \lambda t} d \lambda
$$

and

$$
h_{s}(x, t) \leq C s^{-\alpha-2} e^{-\frac{A}{s}|(x, t)|^{2}},
$$

where $A$ is a constant.

Let $\mathcal{S}(\mathbf{K})$ be the Schwartz space of functions $\psi: \mathbf{R}^{2} \rightarrow \mathbf{C}$ even with respect to the first variable, $\mathcal{C}^{\infty}$ on $\mathbf{R}^{2}$ and rapidly decreasing together with all their derivatives, i.e., for all $k, p, q \in \mathbf{N}$ we have

$$
\widetilde{\mathcal{N}_{k, p, q}}(\psi)=\sup _{(x, t) \in \mathbf{K}}\left\{\left(1+x^{2}+t^{2}\right)^{k}\left|\frac{\partial^{p+q}}{\partial x^{p} \partial t^{q}} \psi(x, t)\right|\right\}<\infty .
$$

Assume $\Psi$ is a function defined on $\mathbf{R} \times \mathbf{N}$. Then let $\Delta_{-} \Psi(\lambda, 0)=\Psi(\lambda, 0)$ and for $m \geq 1$,

$$
\Delta_{-} \Psi(\lambda, m)=\Psi(\lambda, m)-\Psi(\lambda, m-1) .
$$

We write

$$
\Delta_{+} \Psi(\lambda, m)=\Psi(\lambda, m+1)-\Psi(\lambda, m),
$$


then we define the following differential operators:

$$
\Lambda_{1} \Psi(\lambda, m)=\frac{1}{|\lambda|}\left(m \Delta_{+} \Delta_{-} \Psi(\lambda, m)+(\alpha+1) \Delta_{+} \Psi(\lambda, m)\right)
$$

and

$$
\Lambda_{2} \Psi(\lambda, m)=\frac{-1}{2 \lambda}\left((\alpha+m+1) \Delta_{+} \Psi(\lambda, m)+m \Delta_{-} \Psi(\lambda, m)\right) .
$$

$\mathcal{S}(\mathbf{R} \times \mathbf{N})$ is the space of functions $\Psi: \mathbf{R} \times \mathbf{N} \rightarrow \mathbf{C}$ satisfying

(i) for all $m, p, q, r, s \in \mathbf{N}$, the function

$$
\lambda \mapsto \lambda^{p}\left(|\lambda|\left(m+\frac{\alpha+1}{2}\right)\right)^{q} \Lambda_{1}^{r}\left(\Lambda_{2}+\frac{\partial}{\partial \lambda}\right)^{s} \Psi(\lambda, m)
$$

is bounded and continuous on $\mathbf{R}, \mathcal{C}^{\infty}$ on $\mathbf{R}$ and such that the left and the right derivatives at zero exist;

(ii) for all $k, p, q \in \mathbf{N}$, we have

$$
\mathcal{V}_{k, p, q}(\Psi)=\sup _{(\lambda, m) \in \mathbf{R} \times \mathbf{N}}\left\{\left(1+\lambda^{2}\left(1+m^{2}\right)\right)^{k}\left|\Lambda_{1}^{p}\left(\Lambda_{2}+\frac{\partial}{\partial \lambda}\right)^{q} \Psi(\lambda, m)\right|\right\}<\infty .
$$

$\mathcal{D}(\mathbf{R} \times \mathbf{N})$ is the subspace of $\mathcal{S}(\mathbf{R} \times \mathbf{N})$ of functions $\Psi$ satisfying the following:

(i) there exists $m_{0} \in \mathbf{N}$ such that $\Psi(\lambda, m)=0$, for all $(\lambda, m) \in \mathbf{R} \times \mathbf{N}$ such that $m>m_{0}$.

(ii) for all $m<m_{0}$, the function $\lambda \mapsto \Psi(\lambda, m)$ is $\mathcal{C}^{\infty}$ on $\mathbf{R}$, with compact support and vanishes out of a neighborhood of zero.

In the following, we introduce some basic notation about the real and complex interpolation, more about these can be found in [3].

The real interpolation includes $K$-method and $J$-method. We first give the $K$-method as follows: let $X$ and $Y$ be two Banach spaces, then for any $u \in X+Y$, denote

$$
K(t, u)=\min _{u=u_{1}+u_{2}}\left(\left\|u_{1}\right\|_{X}+t\left\|u_{2}\right\|_{Y}\right)
$$

and

$$
\|u\|_{\theta, q ; K}=\left(\int_{0}^{\infty} t^{-\theta}(K(t, u))^{q} \frac{d t}{t}\right)^{1 / q}
$$

where $1 \leq q \leq \infty$. The $K$-method of real interpolation consists in taking $K_{\theta, q}(X, Y)$ to be the set of all $u$ in $X+Y$ such that $\|u\|_{\theta, q ; K}<\infty$.

The $J$-method of real interpolation is defined as follows: for any $u \in X \cap Y$, let

$$
J(t, u)=\max \left(\|u\|_{X}, t\|u\|_{Y}\right)
$$

Then, $u$ is in $J_{\theta, q}(X, Y)$ if and only if it can be written as

$$
u=\int_{0}^{\infty} v(t) \frac{d t}{t}
$$


where $v(t)$ is measurable with value in $X \cap Y$ and such that

$$
\Phi(v)=\left(\int_{0}^{\infty} t^{-\theta q}(J(t, v(t)))^{q} \frac{d t}{t}\right)^{1 / q}<\infty .
$$

The norm of $u$ is $\|u\|_{\theta, q ;}:=\inf _{v} \Phi(v)$.

The complex interpolation consists in looking at the space of analytic functions $f$ with values in $X+Y$ defined on the open strip $0<\Re Z<1$ and continuous on the closed strip $0 \leq \Re Z \leq 1$, and such that $f(i y)$ is bounded in $X, f(1+i y)$ is bounded in $Y$. We define the norm

$$
\|f\|=\max \left\{\sup _{y}\|f(i y)\|_{X}, \sup _{y}\|f(1+i y)\|_{Y}\right\} .
$$

For $0<\theta<1$, one defines $[X, Y]_{\theta}=\{u \in X+Y\}$, with the norm $\|u\|=\inf _{f(\theta)=u}\|f\|$.

The paper is organized as follows. In Section 2, we will investigate Sobolev spaces on K. A Besov space and a Triebel-Lizorkin space will be studied in Section 3 and Section 4 respectively.

Throughout the paper, we will use $C$ to denote the positive constant, which is not necessarily the same at each occurrence.

\section{Sobolev spaces on $\mathrm{K}$}

In this section, we will study a Bessel potential space on the Laguerre hypergroup $\mathbf{K}$.

Let $s \in \mathbf{R}$. Then the Bessel potential on $\mathbf{K}$ is defined by

$$
J^{s}=(I+L)^{\frac{-s}{2}}=\Gamma\left(\frac{s}{2}\right) \int_{0}^{+\infty} t^{\left(\frac{s}{2}\right)-1} e^{-t} e^{-t L} d t
$$

It is easy to prove that the Bessel potentials satisfy the following semigroup property: $J^{s} J^{t}=$ $J^{s+t}$ and $J^{-m} J^{s}=J^{s-m}$, where $s, t \in \mathbf{R}, m \in \mathbf{N}$ and $s>m$.

The Bessel potentials also satisfy the following property.

Proposition 2 The Bessel potential $J^{s}: L_{\alpha}^{p}(\mathbf{K}) \rightarrow L_{\alpha}^{p}(\mathbf{K})$ is bounded, where $s>0$ and $1 \leq$ $p \leq \infty$

Proof Let $1 \leq p \leq \infty$ and $f \in L_{\alpha}^{p}(\mathbf{K})$. Then

$$
\left(J^{s} f\right)(x, r)=\Gamma\left(\frac{s}{2}\right) \int_{\mathbf{K}} \int_{0}^{+\infty} t^{\left(\frac{s}{2}\right)-1} e^{-t} T_{(x, r)}^{(\alpha)} h_{t}(y, l) f(y,-l) d t d m_{\alpha}(y, l) .
$$

Since

$$
\begin{aligned}
& \Gamma\left(\frac{s}{2}\right) \int_{\mathbf{K}} \int_{0}^{+\infty} t^{\left(\frac{s}{2}\right)-1} e^{-t} T_{(x, r)}^{(\alpha)} h_{t}(y, l) d t d m_{\alpha}(x, r) \\
& \quad=\Gamma\left(\frac{s}{2}\right) \int_{0}^{+\infty} t^{\left(\frac{s}{2}\right)-1} e^{-t} \int_{\mathbf{K}} T_{(x, r)}^{(\alpha)} h_{t}(y, l) d m_{\alpha}(x, r) d t \\
& \quad \leq \Gamma\left(\frac{s}{2}\right) \int_{0}^{+\infty} t^{\left(\frac{s}{2}\right)-1} e^{-t} \int_{\mathbf{K}} h_{t}(y, l) d m_{\alpha}(x, r) d t \\
& \quad \leq 1
\end{aligned}
$$


$J^{s}: L_{\alpha}^{p}(\mathbf{K}) \rightarrow L_{\alpha}^{p}(\mathbf{K})$ is bounded for $s>0$ and $1 \leq p \leq \infty$ follows from (6). This gives the proof of Proposition 2.

Now, we define the Bessel potential space on $\mathbf{K}$.

Definition 1 For $1 \leq p \leq \infty, s \in \mathbf{R}$, we define the Bessel potential space $W_{p}^{s}(\mathbf{K})$ as follows: If $s>0$, then $W_{p}^{s}(\mathbf{K})$ is the collection of all functions $f \in L_{\alpha}^{p}(\mathbf{K})$ such that $f=J^{s} h$ for some $h \in L_{\alpha}^{p}(\mathbf{K})$ with the norm $\|f\|_{W_{p}^{s}}=\|h\|_{\alpha, p}$;

If $s<0$, then $W_{p}^{s}(\mathbf{K})$ is the collection of all distributions $f \in \mathcal{S}^{\prime}(\mathbf{K})$ such that $f=J^{-2 m} h$ for some $h \in W_{p}^{2 m+s}(\mathbf{K})$, where $m \in \mathbf{N}$ with $2 m+s>0$, and $\|f\|_{W_{p}^{s}}=\|h\|_{W_{p}^{2 m+s}}$;

If $s=0$, then $W_{p}^{0}(\mathbf{K})=L_{\alpha}^{p}(\mathbf{K})$.

\section{Remark 1}

(1) When $s>0$ and $1<p<\infty$, we call $W_{p}^{s}(\mathbf{K})$ the Sobolev space on $\mathbf{K}$.

(2) It is easy to know that the definition of the space $W_{p}^{s}(\mathbb{K})$ with $s<0$ is independent of $m$.

In the following, we prove that the spaces $W_{p}^{s}(\mathbf{K})$ are complete.

Proposition 3 The Bessel potential spaces $W_{p}^{s}(\mathbf{K})$, where $1 \leq p \leq \infty$ and $s \in \mathbf{R}$, are complete.

Proof If $s>0$, let $\left\{f_{n}\right\}$ be a Cauchy sequence in $W_{p}^{s}(\mathbf{K})$, then $\left\{J^{-s} f_{n}\right\}$ is a Cauchy sequence in $L_{\alpha}^{p}(\mathbf{K})$. So there exists $g \in L_{\alpha}^{p}(\mathbf{K})$ such that

$$
\left\|J^{-s} f_{n}-g\right\|_{\alpha, p} \rightarrow 0, \quad n \rightarrow \infty
$$

Therefore,

$$
\left\|f_{n}-J^{s} g\right\|_{W_{p}^{s}}=\left\|J^{-s} f_{n}-g\right\|_{\alpha, p} \rightarrow 0, \quad n \rightarrow \infty
$$

By Proposition 2, $J^{s} g \in L_{\alpha}^{p}(\mathbf{K})$. This proves that $W_{p}^{s}(\mathbf{K})$ is complete with $s>0$ and $1 \leq p \leq \infty$.

If $s<0$, let $\left\{f_{n}\right\}$ be a Cauchy sequence in $W_{p}^{s}(\mathbf{K})$, then there exists a sequence $\left\{h_{n}\right\}$ in $W_{p}^{2 m+s}(\mathbf{K})$ such that $f_{n}=J^{-2 m} h_{n}$ and $\left\|f_{n}\right\|_{W_{p}^{s}}=\left\|h_{n}\right\|_{W_{p}^{2 m+s}}$. Therefore, $\left\{h_{n}\right\}$ is a Cauchy sequence in $W_{p}^{2 m+s}(\mathbf{K})$. Following from the case of $s>0$, there exists $h \in W_{p}^{2 m+s}(\mathbf{K})$ such that

$$
\left\|h_{n}-h\right\|_{W_{p}^{2 m+s}} \rightarrow 0, \quad n \rightarrow \infty
$$

Since $h \in W_{p}^{2 m+s}(\mathbf{K})$, we have $J^{-2 m} h \in W_{p}^{s}(\mathbf{K})$ and

$$
\left\|f_{n}-J^{-2 m} h\right\|_{W_{p}^{s}}=\left\|J^{-2 m} h_{n}-J^{-2 m} h\right\|_{W_{p}^{s}}=\left\|h_{n}-h\right\|_{W_{p}^{2 m+s}} \rightarrow 0, \quad n \rightarrow \infty .
$$

Therefore, $W_{p}^{s}(\mathbf{K})$ is complete with $s<0$.

If $s=0$, the result is obvious. This completes the proof of Proposition 3 .

The Bessel potential space satisfies: 
Proposition 4 Let $s, t \in \mathbf{R}$ and $1 \leq p \leq \infty$, we have

(1) If $s>t$, then $W_{p}^{s}(\mathbf{K}) \subseteq W_{p}^{t}(\mathbf{K})$;

(2) $J^{s}: W_{p}^{t}(\mathbf{K}) \rightarrow W_{p}^{s+t}(\mathbf{K})$ is an isomorphism;

(3) $\left(W_{p}^{s}(\mathbf{K})\right)^{\prime}=W_{p^{\prime}}^{-s}(\mathbf{K})$, where $\frac{1}{p}+\frac{1}{p^{\prime}}=1$.

Proof We will give the proof of the case $s>0$, the other cases can be proved similarly.

(1) Let $f \in W_{p}^{s}(\mathbf{K})$. Then there exists $h \in L_{\alpha}^{p}(\mathbf{K})$ such that

$$
f=(I+L)^{-\frac{s}{2}} h=(I+L)^{-\frac{t}{2}}(I+L)^{-\frac{s-t}{2}} h .
$$

Since $s>t$, by Proposition $2, J^{s-t}$ is bounded on $L_{\alpha}^{p}(\mathbf{K})$. Therefore, $(I+L)^{-\frac{s-t}{2}} h \in L_{\alpha}^{p}(\mathbf{K})$, then $f \in W_{p}^{t}(\mathbf{K})$. This proves $W_{p}^{s}(\mathbf{K}) \subseteq W_{p}^{t}(\mathbf{K})$.

(2) For $f \in W_{p}^{t}(\mathbf{K})$, there exists $h \in L_{\alpha}^{p}(\mathbf{K})$ such that $f=(I+L)^{-\frac{t}{2}} h$. Therefore,

$$
J^{s} f=(I+L)^{-\frac{s}{2}} f=(I+L)^{-\frac{s+t}{2}} h \in W_{p}^{s+t}(\mathbf{K})
$$

and

$$
\left\|J^{s} f\right\|_{W_{p}^{s+t}}=\|h\|_{\alpha, p}=\|f\|_{W_{p}^{t}} .
$$

(3) For $f \in W_{p}^{s}(\mathbf{K})$ and $g \in W_{p^{\prime}}^{-s}(\mathbf{K})$, there exist $h_{1} \in L_{\alpha}^{p}(\mathbf{K}), h_{2} \in W_{p^{\prime}}^{2 m-s}(\mathbf{K})$ such that $f=$ $J^{s} h_{1}, g=J^{-2 m} h_{2}$. Since $h_{2}=J^{2 m-s} h_{3}$, where $h_{3} \in L_{\alpha}^{p^{\prime}}(\mathbf{K})$, we have

$$
\langle f, g\rangle=\left\langle J^{s} h_{1}, J^{-2 m} h_{2}\right\rangle=\left\langle J^{s} h_{1}, J^{-s} h_{3}\right\rangle .
$$

By the part (2) that we have proved, we have

$$
\langle f, g\rangle=\left\langle J^{s} h_{1}, J^{-s} h_{3}\right\rangle=\left\langle J^{2 s} h_{1}, h_{3}\right\rangle .
$$

Note $J^{2 s} h_{1} \in L_{\alpha}^{p}(\mathbf{K}), h_{3} \in L_{\alpha}^{p^{\prime}}(\mathbf{K})$, we can get $W_{p^{\prime}}^{-s}(\mathbf{K}) \subseteq\left(W_{p}^{s}(\mathbf{K})\right)^{\prime}$.

For the reverse, let $T \in\left(W_{p}^{s}(\mathbf{K})\right)^{\prime}$, then there exists $C>0$ such that

$$
|T f| \leq C\|f\|_{W_{p}^{s}}, \quad f \in W_{p}^{s}(\mathbf{K}) .
$$

For any $h \in L_{\alpha}^{p}(\mathbf{K})$, let $f=J^{s} h$, then $\left|T J^{s} h\right| \leq C\|h\|_{\alpha, p}$, i.e., $T J^{s} \in\left(L_{\alpha}^{p}(\mathbf{K})\right)^{\prime}$. Therefore, there exists $g \in L_{\alpha}^{p^{\prime}}(\mathbf{K})$, such that

$$
\begin{aligned}
T J^{s} h & =\int_{\mathbf{K}} h(x, t) \bar{g}(x, t) d m_{\alpha}(x, t)=\int_{\mathbf{K}} J^{s} h(x, t) J^{s} \bar{g}(x, t) d m_{\alpha}(x, t) \\
& =\int_{\mathbf{K}} f(x, t) J^{s} \bar{g}(x, t) d m_{\alpha}(x, t)=\int_{\mathbf{K}} f(x, t) J^{-s} J^{2 s} \bar{g}(x, t) d m_{\alpha}(x, t) .
\end{aligned}
$$

Since $J^{2 s} \bar{g} \in L_{\alpha}^{p^{\prime}}(\mathbf{K})$, let $k=J^{-s}\left(J^{2 s} g\right) \in W_{p^{\prime}}^{-s}(\mathbf{K})$, then

$$
T f=\int_{\mathbf{K}} f(x, t) \bar{k}(x, t) d m_{\alpha}(x, t) .
$$

Therefore, $\left(W_{p}^{s}(\mathbf{K})\right)^{\prime} \subseteq W_{p^{\prime}}^{-s}(\mathbf{K})$. We complete the proof of Proposition 4 . 


\section{Besov space on $\mathrm{K}$}

In this section, we will define a Besov space on $\mathbf{K}$ by the real interpolation of the Bessel potential spaces.

Definition $2 \rho \in L^{1}(\mathbf{R} \times \mathbf{N})$ is called a Fourier multiplier on $L_{\alpha}^{p}(\mathbf{K})$ if the convolution $\left(\mathcal{F}^{-1} \rho\right) * f \in L_{\alpha}^{p}(\mathbf{K})$ for all $f \in L_{\alpha}^{p}(\mathbf{K})$ and

$$
\|\rho\|_{M_{p}}=\sup _{\|f\|_{\alpha, p}=1}\left\|\left(\mathcal{F}^{-1} \rho\right) * f\right\|_{\alpha, p}<\infty
$$

where $1 \leq p \leq \infty$. The linear space of all such $\rho$ is denoted by $M_{p}$, the norm on $M_{p}$ is $\|\cdot\|_{M_{p}}$.

We have the following property about the Fourier multiplier on $L_{\alpha}^{p}(\mathbf{K})$.

Proposition 5 If $\rho \in M_{p}$, then $\rho_{r} \in M_{p}$ and $\left\|\rho_{r}\right\|_{M_{p}}=\|\rho\|_{M_{p}}$, where $1 \leq p \leq \infty$ and $\rho_{r}(\lambda, m)=\rho\left(r^{2} \lambda, m\right)$.

Proof It is easy to prove

$$
\left(\mathcal{F}^{-1} \rho_{r}\right) * f(x, t)=r^{-(2 \alpha+4)}\left(\mathcal{F}^{-1} \rho\right) * f_{\frac{1}{r}}\left(\frac{x}{r}, \frac{t}{r^{2}}\right) .
$$

Therefore,

$$
\left\|\left(\mathcal{F}^{-1} \rho_{r}\right) * f\right\|_{\alpha, p}=r^{-\frac{(2 \alpha+4)(p-1)}{p}}\left\|\left(\mathcal{F}^{-1} \rho\right) * f_{\frac{1}{r}}\right\|_{\alpha, p} .
$$

By $\left\|f_{\frac{1}{r}}\right\|_{\alpha, p}=r^{\frac{(2 \alpha+4)(p-1)}{p}}\|f\|_{\alpha, p}$, we get

$$
\begin{aligned}
\left\|\rho_{r}\right\|_{M_{p}} & =\sup _{\|f\|_{\alpha, p}=1}\left\|\left(\mathcal{F}^{-1} \rho_{r}\right) * f\right\|_{\alpha, p}=r^{-\frac{(2 \alpha+4)(p-1)}{p}} \sup _{\|f\|_{\alpha, p}=1}\left\|\left(\mathcal{F}^{-1} \rho\right) * f_{\frac{1}{r}}\right\|_{\alpha, p} \\
& =\sup _{\|g\|_{\alpha, p}=1}\left\|\left(\mathcal{F}^{-1} \rho\right) * g\right\|_{\alpha, p}=\|\rho\|_{M_{p}},
\end{aligned}
$$

where $g=r^{-\frac{(2 \alpha+4)(p-1)}{p}} f_{\frac{1}{r}}$. This proves Proposition 5 .

Let $\Phi \in \mathcal{D}(\mathbf{R} \times \mathbf{N})$ satisfy supp $\Phi=\left\{(\lambda, m): 2^{-2} \leq|\lambda| \leq 4, m \leq m_{0}\right\}$ and $\varphi(\lambda, m)>0$, for $2^{-2}<|\lambda|<4, m<m_{0}$. Then, for $m<m_{0}$, let

$$
\varphi(\lambda, m)=\frac{\Phi(\lambda, m)}{\sum_{k=-\infty}^{+\infty} \Phi\left(2^{-k} \lambda, m\right)}
$$

and $\varphi(\lambda, m)=0$ for $m \geq m_{0}$, we have

(i) $\operatorname{supp} \varphi=\left\{(\lambda, m): 2^{-2} \leq|\lambda| \leq 4, m \leq m_{0}\right\}$;

(ii) $\varphi(\lambda, m)>0$, for $2^{-2}<|\lambda|<4, m<m_{0}$;

(iii) $\sum_{k=-\infty}^{+\infty} \varphi\left(2^{-2 k} \lambda, m\right)=1$, for $\lambda \neq 0, m<m_{0}$.

We define functions $\varphi_{k}$ and $\psi$ on $\mathbf{K}$ by $\left(\mathcal{F} \varphi_{k}\right)(\lambda, m)=\varphi\left(2^{-2 k} \lambda, m\right),(\mathcal{F} \psi)(\lambda, m)=1-$ $\sum_{k=1}^{\infty} \varphi\left(2^{-2 k} \lambda, m\right)$. Then, we have: 
Lemma 2 Let $f \in \mathcal{S}^{\prime}(\mathbf{K})$ and assume $\varphi_{k} * f \in L_{\alpha}^{p}(\mathbf{K})$, where $1 \leq p \leq \infty$ and $s \in \mathbf{R}$. Then

$$
\left\|J^{s} \varphi_{k} * f\right\|_{\alpha, p} \leq C 2^{-s k}\left\|\varphi_{k} * f\right\|_{\alpha, p}
$$

where $k \geq 1$.

$$
\text { If } \psi * f \in L_{\alpha}^{p}(\mathbf{K}) \text {, then }
$$

$$
\left\|J^{s} \psi * f\right\|_{\alpha, p} \leq C\|\psi * f\|_{\alpha, p} .
$$

Proof For $k \in \mathbf{N}$, we have

$$
\varphi_{k} * f=\sum_{l=-1}^{l=1}\left(\varphi_{k+l} * \varphi_{k} * f\right)
$$

Therefore, it is sufficient to prove that

$$
\left\|\mathcal{F}\left(J^{s} \varphi_{k+l}\right)\right\|_{M_{p}} \leq 2^{-k s}
$$

By

$$
\mathcal{F}\left(J^{s} \varphi_{k+l}\right)(\lambda, m)=(1+|\lambda|(2 \alpha+2+4 m))^{-\frac{s}{2}} \varphi\left(2^{-2(k+l)} \lambda, m\right)
$$

and Proposition 5, we know that the above function has the same norm in $M_{p}$ as the function

$$
2^{-(k+l) s}\left(2^{-2(k+l)}+|\lambda|(2 \alpha+2+4 m)\right)^{-\frac{s}{2}} \varphi(\lambda, m) .
$$

Then Lemma 2 gives

$$
\left\|2^{-(k+l) s}\left(2^{-2(k+l)}+|\lambda|(2 \alpha+2+4 m)\right)^{-\frac{s}{2}} \varphi(\lambda, m)\right\|_{M_{p}} \leq C 2^{-k s} .
$$

Since $\psi * f=\left(\psi+\varphi_{1}\right) * \psi * f$, we just need to prove $\mathcal{F}\left(J^{s} \psi\right) \in M_{p}$. Let $l>\frac{\alpha+2}{4}$. Then

$$
\begin{aligned}
& \int_{\mathbf{K}}\left|J^{s} \psi(x, t)\right| d m_{\alpha}(x, t) \\
& \quad=\int_{\|(x, t)\|>1}\left|J^{s} \psi(x, t)\right| d m_{\alpha}(x, t)+\int_{\|(x, t)\| \leq 1}\left|J^{s} \psi(x, t)\right| d m_{\alpha}(x, t) \\
& \quad=I_{1}+I_{2} .
\end{aligned}
$$

Let $\Lambda=\Lambda_{1}+2\left(\Lambda_{2}+\frac{\partial}{\partial \lambda}\right)$, then by the Hölder inequality,

$$
\begin{aligned}
I_{1} & =\int_{\|(x, t)\|>1}\left(x^{4}+4 t^{2}\right)^{-l}\left(x^{4}+4 t^{2}\right)^{l}\left|J^{s} \psi(x, t)\right| d m_{\alpha}(x, t) \\
& \leq\left(\int_{\|(x, t)\|>1}\left(x^{4}+4 t^{2}\right)^{-2 l} d m_{\alpha}(x, t)\right)^{\frac{1}{2}}\left(\int_{\|(x, t)\|>1}\left(x^{4}+4 t^{2}\right)^{2 l}\left|J^{s} \psi(x, t)\right|^{2} d m_{\alpha}(x, t)\right)^{\frac{1}{2}} \\
& =\left(\int_{\|(x, t)\|>1}\left(x^{4}+4 t^{2}\right)^{-2 l} d m_{\alpha}(x, t)\right)^{\frac{1}{2}}\left(\int_{\|(x, t)\|>1}\left|\mathcal{F}^{-1}\left(\Lambda^{l} \mathcal{F}\left(J^{s} \psi\right)\right)(x, t)\right|^{2} d m_{\alpha}(x, t)\right)^{\frac{1}{2}} .
\end{aligned}
$$


Since $l>\frac{\alpha+2}{4}$, by the Plancherel theorem,

$$
I_{1} \leq C\left(\int_{\mathbf{R} \times \mathbf{N}}\left|\left(\Lambda^{l} \mathcal{F}\left(J^{s} \psi\right)\right)(\lambda, m)\right|^{2} d \gamma_{\alpha}(\lambda, m)\right)^{\frac{1}{2}}
$$

Since $\Lambda^{l} \mathcal{F}\left(J^{s} \psi\right) \in L_{\alpha}^{2}(\mathbf{R} \times \mathbf{N})$, we get $I_{1}<\infty$.

Now, we estimate $I_{2}$,

$$
\begin{aligned}
I_{2} & \leq\left(\int_{\|(x, t)\| \leq 1} d m_{\alpha}(x, t)\right)^{\frac{1}{2}}\left(\int_{\|(x, t)\| \leq 1}\left|J^{s} \psi(x, t)\right|^{2} d m_{\alpha}(x, t)\right)^{\frac{1}{2}} \\
& \leq C\left(\int_{\mathbf{R} \times \mathbf{N}}\left|\mathcal{F}\left(J^{s} \psi\right)(\lambda, m)\right|^{2} d \gamma_{\alpha}(\lambda, m)\right)^{\frac{1}{2}} .
\end{aligned}
$$

Since $\mathcal{F}\left(J^{s} \psi\right) \in L_{\alpha}^{2}(\mathbf{R} \times \mathbf{N})$, we get $I_{2}<\infty$. Therefore, $J^{s} \psi \in L_{\alpha}^{1}(\mathbf{K})$ and Lemma 2 is proved.

Definition 3 For $s \in \mathbf{R}, 1 \leq p \leq \infty$ and $1 \leq q \leq \infty$, we define the Besov space $B_{p, q}^{s}(\mathbf{K})$ as

$$
B_{p, q}^{s}(\mathbf{K})=\left\{f \in \mathcal{S}^{\prime}(\mathbf{K}):\|f\|_{p, q}^{s}<\infty\right\},
$$

where

$$
\|f\|_{p, q}^{s}=\|\psi * f\|_{\alpha, p}+\left(\sum_{k=1}^{\infty}\left(2^{s k}\left\|\varphi_{k} * f\right\|_{\alpha, p}\right)^{q}\right)^{\frac{1}{q}} .
$$

Remark 2 By Theorem 3.4.2 in [3], we know $B_{p, q}^{s}(\mathbf{K})$ is complete with $s \in \mathbf{R}, 1 \leq p \leq \infty$ and $1 \leq q \leq \infty$.

In the following, we prove that our definition coincides with Definition 4.1 in [1] for $s>0,1 \leq p \leq \infty$ and $1 \leq q \leq \infty$.

Theorem 1 Let $1 \leq p, q \leq \infty, s \in \mathbf{R}$. Then we have

$$
B_{p, q}^{s}(\mathbf{K})=K_{\theta, q}\left(W_{p}^{s_{0}}(\mathbf{K}), W_{p}^{s_{1}}(\mathbf{K})\right)
$$

where $s=(1-\theta) s_{0}+\theta s_{1}, 0<\theta<1, s_{0}, s_{1} \in \mathbf{R}$ and $s_{0} \neq s_{1}$

Proof Let $f \in K_{\theta, q}\left(W_{p}^{s_{0}}(\mathbf{K}), W_{p}^{s_{1}}(\mathbf{K})\right)$ and put $f=f_{0}+f_{1}, f_{i} \in W_{p}^{s_{i}}(i=0,1)$. By Lemma 2,

$$
\begin{aligned}
\left\|\varphi_{k} * f\right\|_{\alpha, p} & \leq\left\|\varphi_{k} * f_{0}\right\|_{\alpha, p}+\left\|\varphi_{k} * f_{1}\right\|_{\alpha, p} \\
& \leq C\left(2^{-s_{0} k}\left\|J^{-s_{0}} f_{0}\right\|_{\alpha, p}+2^{-s_{1} k}\left\|J^{-s_{1}} f_{1}\right\|_{\alpha, p}\right) .
\end{aligned}
$$

So

$$
\left\|\varphi_{k} * f\right\|_{\alpha, p} \leq C 2^{-s_{0} k} K\left(2^{\left(s_{0}-s_{1}\right) k}, f\right) .
$$


This shows

$$
\left(\sum_{k=1}^{\infty}\left(2^{s k}\left\|\varphi_{k} * f\right\|_{\alpha, p}\right)^{q}\right)^{\frac{1}{q}} \leq C\|f\|_{\theta, q ; K} .
$$

Similarly, we can prove

$$
\|\psi * f\|_{\alpha, p} \leq C K(1, f) \leq C\|f\|_{\theta, q ; K} .
$$

Therefore,

$$
\|f\|_{p, q}^{s} \leq C\|f\|_{\theta, q ; K}
$$

This proves

$$
B_{p, q}^{s}(\mathbf{K}) \subseteq K_{\theta, q}\left(W_{p}^{s_{0}}(\mathbf{K}), W_{p}^{s_{1}}(\mathbf{K})\right)
$$

By Lemma 2 again, we have

$$
2^{\left(s-s_{0}\right) k} J\left(2^{\left(s_{0}-s_{1}\right) k}, \varphi_{k} * f\right) \leq C 2^{s k}\left\|\varphi_{k} * f\right\|_{\alpha, p}
$$

and

$$
J\left(1, \varphi_{k} * f\right) \leq C\|\psi * f\|_{\alpha, p},
$$

where

$$
f \in K_{\theta, q}\left(W_{p}^{s_{0}}(\mathbf{K}), W_{p}^{s_{1}}(\mathbf{K})\right) .
$$

By Lemma 3.2.1 in [3], we know that $J(t, f)$ is increasing with respect to $t$. Therefore,

$$
\begin{aligned}
\|f\|_{\theta, q, J} & =\left(\int_{0}^{\infty}\left(t^{-\theta} J(t, f)\right)^{q} \frac{d t}{t}\right)^{\frac{1}{q}} \\
& \leq J\left(1, \varphi_{k} * f\right)+\left(\sum_{k=1}^{\infty}\left(2^{\left(s-s_{0}\right) k} J\left(2^{\left(s_{0}-s_{1}\right) k}, \varphi_{k} * f\right)\right)^{q}\right)^{\frac{1}{q}} \\
& \leq C\|\psi * f\|_{\alpha, p}+C\left(\sum_{k=1}^{\infty}\left(2^{s k}\left\|\varphi_{k} * f\right\|_{\alpha, p}\right)^{q}\right)^{\frac{1}{q}} \\
& =C\|f\|_{p, q}^{s}<\infty .
\end{aligned}
$$

By Theorem 3.3.1 in [3], we know that $J(t, f)$ is equivalent to $K(t, f)$. So

$$
\|f\|_{\theta, q ; K} \leq C\|f\|_{\theta, q, J}<\infty
$$


Since

$$
f=\psi * f+\sum_{k=1}^{\infty} \varphi_{k} * f
$$

it is sufficient to prove

$$
\psi * f+\sum_{k=1}^{\infty} \varphi_{k} * f \in W_{p}^{s_{0}}(\mathbf{K})+W_{p}^{s_{1}}(\mathbf{K})
$$

Assume $s_{0}<s_{1}$, then

$$
W_{p}^{s_{0}}(\mathbf{K})+W_{p}^{s_{1}}(\mathbf{K})=W_{p}^{s_{0}}(\mathbf{K}) .
$$

By Lemma 2, we have

$$
\begin{aligned}
\|f\|_{W_{p}^{s 0}} & \leq\|\psi * f\|_{W_{p}^{s_{0}}}+\left\|\sum_{k=1}^{\infty} \varphi_{k} * f\right\|_{W_{p}^{s_{0}}} \\
& \leq C\left(\|\psi * f\|_{\alpha, p}+\sum_{k=1}^{\infty} 2^{\left(s_{0}-s\right) k} 2^{k s}\left\|\varphi_{k} * f\right\|_{\alpha, p}\right) \\
& \leq C\|f\|_{p, q}^{s}<\infty .
\end{aligned}
$$

This gives the proof of Theorem 1 .

We have the following version of the Calderón reproducing formula on $\mathbf{K}$, the proof is standard (cf. [6]).

Lemma 3 Let $\varphi \in \mathcal{S}(\mathbf{K})$ and satisfy

$$
\int_{\mathbf{K}} \varphi(x, t) d m_{\alpha}(x, t)=0
$$

For $f \in \mathcal{S}^{\prime}(\mathbf{K})$ satisfying

$$
f * \varphi_{r} \rightarrow 0 \quad \text { in } \mathcal{S}^{\prime}(\mathbf{K}), \text { as } r \rightarrow+\infty
$$

we have

$$
\int_{\epsilon}^{A} f * \varphi_{r} * \varphi_{r}(x, t) \frac{d r}{r} \rightarrow f(x, t) \quad \text { in } \mathcal{S}^{\prime}(\mathbf{K}),
$$

when $\epsilon \rightarrow 0$ and $A \rightarrow+\infty$.

Remark 3 When $f \in L_{\alpha}^{p}(\mathbf{K})$ for $1 \leq p \leq+\infty$, it is easy to prove that $f$ satisfies the condition of Lemma 3. 
Theorem 2 Let $1 \leq p, q \leq \infty$ and $s>0$. Then

$$
L_{\alpha}^{p}(\mathbf{K}) \cap \dot{\Lambda}_{p, q}^{s}(\mathbf{K})=B_{p, q}^{s}(\mathbf{K})
$$

where $\dot{\Lambda}_{p, q}^{s}(\mathbf{K})$ is the generalized homogeneous Besov-Laguerre type space defined in [1].

Proof By the Theorem 3.13 in [1],

$$
L_{\alpha}^{p}(\mathbf{K}) \cap \dot{\Lambda}_{p, q}^{s}(\mathbf{K}) \subseteq B_{p, q}^{s}(\mathbf{K}) .
$$

Conversely, let $f \in B_{p, q}^{s}(\mathbf{K})$, then by Lemma 2,

$$
\left\|\varphi_{k} * f\right\|_{\alpha, p} \leq C\|\psi * f\|_{\alpha, p}, \quad k<0 .
$$

Thus, for $s>0$,

$$
\left(\sum_{k<0}\left(2^{s k}\left\|\varphi_{k} * f\right\|_{\alpha, p}\right)^{q}\right)^{\frac{1}{q}} \leq C\|\psi * f\|_{\alpha, p} .
$$

When $s>0$, it is easy to prove $f \in L_{\alpha}^{p}(\mathbf{K})$ for $f \in B_{p, q}^{s}(\mathbf{K})$. By Remark 3 and Lemma 3, we have $f \in \dot{\Lambda}_{p, q}^{s}(\mathbf{K})$. Therefore,

$$
L_{\alpha}^{p}(\mathbf{K}) \cap \dot{\Lambda}_{p, q}^{s}(\mathbf{K})=B_{p, q}^{s}(\mathbf{K}) .
$$

This completes the proof of Theorem 2 .

By Theorem 1 and Theorem 2, we know our definition coincides with the Definition 4.1 in [1] for $1 \leq p, q \leq \infty$ and $s>0$.

By the properties of the Bessel potential space and the real interpolation, we can get the following properties about the Besov space, which are similar to those of the classical Besov space.

\section{Proposition 6}

(1) If $s_{1}<s_{2}$, then $B_{p, q}^{s_{2}}(\mathbf{K}) \subset B_{p, q}^{s_{1}}(\mathbf{K}), s_{1}, s_{2} \in \mathbf{R}, 1 \leq p, q \leq \infty$.

(2) If $1 \leq q_{1}<q_{2} \leq \infty$, then $B_{p, q_{1}}^{s}(\mathbf{K}) \subset B_{p, q_{2}}^{s}(\mathbf{K})$, where $s \in \mathbf{R}, 1 \leq p \leq \infty$.

(3) $B_{p, 1}^{s} \subseteq W_{p}^{s} \subset B_{p, \infty}^{s}, s \in \mathbf{R}, 1 \leq p \leq \infty$.

(4) $\left(B_{p, q}^{s}(\mathbf{K})\right)^{\prime}=B_{p^{\prime}, q^{\prime}}^{-s}(\mathbf{K}), s \in \mathbf{R}, 1 \leq p \leq \infty, 1 \leq q<\infty$.

(5) $J^{t}: B_{p, q}^{s}(\mathbf{K}) \rightarrow B_{p, q}^{s+t}(\mathbf{K})$ is a linear bounded one-to-one operator.

(6) $B_{p, q}^{s}(\mathbf{K}) \subset B_{p_{1}, q_{1}}^{s_{1}}(\mathbf{K}), 1 \leq p \leq p_{1} \leq \infty, 1 \leq q \leq q_{1} \leq \infty, s, s_{1} \in \mathbf{R}, s-\frac{2 \alpha+4}{p}=s_{1}-\frac{2 \alpha+4}{p_{1}}$.

\section{Triebel-Lizorkin space on $\mathrm{K}$}

In this section, we will define a Triebel-Lizorkin space on $\mathbf{K}$ by the complex interpolation of the Bessel potential space and the Besov space. Then, we study some basic properties about the Triebel-Lizorkin space on $\mathbf{K}$. 
Definition 4 Let $1<p, q<\infty$ and $s \in \mathbf{R}$. Then the Triebel-Lizorkin space on $\mathbf{K}$ is defined by

$$
F_{p, q}^{s}(\mathbf{K})=\left\{f \in \mathcal{S}^{\prime}(\mathbf{K}):\|f\|_{F_{p, q}^{s}}<\infty\right\}
$$

where

$$
\|f\|_{F_{p, q}^{s}}=\left\|\left(\sum_{j=0}^{\infty}\left|2^{j s} f * \varphi_{j}\right|^{q}\right)^{\frac{1}{q}}\right\|_{\alpha, p} .
$$

In order to give an equivalent norm for $F_{p, q}^{s}(\mathbf{K})$, we need the following Lemma (cf. [8]).

Lemma 4 Let $h(\lambda, m)$ be a $\left(\left[\frac{\alpha+1}{2}\right]+1\right)$ times differentiable function on $\mathbb{R}^{2}$ and satisfy

$$
\left|\left(\Lambda_{1}+2\left(\Lambda_{2}+\frac{\partial}{\partial \lambda}\right)\right)^{j} h(\lambda, m)\right| \leq C_{j}((4 m+2 \alpha+2)|\lambda|)^{-j}
$$

for $j=0,1,2, \ldots,\left[\frac{\alpha+1}{2}\right]+1$, and $T$ be an operator defined by $\widehat{T f}(\lambda, m)=h(\lambda, m) \hat{f}(\lambda, m)$. Then $T$ is bounded on $L_{\alpha}^{p}(\mathbf{K})$, where $1<p<\infty$.

The proof of the following lemma can be found in [5].

Lemma 5 Let $s \in \mathbf{R}$ and $\left\{r_{j}(t)\right\}_{j=0}^{\infty}$ be the Rademacher functions (cf. [10]). Then for every $p$, with $1<p<\infty$ and $t \in[0,1]$, we have constants $A_{1}$, $A_{2}$ such that $\left\|\mathcal{F}^{-1}\left(m_{i} \hat{f}\right)\right\|_{\alpha, p} \leq A_{i}\|f\|_{\alpha, p}$, $i=1,2$,

$$
m_{1}(\lambda, m)=\sum_{j=0}^{\infty} 2^{j s} r_{j}(t)\left(1+4\left(m+\frac{\alpha+1}{2}\right)|\lambda|\right)^{-\frac{s}{2}} \varphi\left(2^{2 j} \lambda, m\right)
$$

and

$$
m_{2}(\lambda, m)=\left(\sum_{j=0}^{\infty} \varphi^{2}\left(2^{2 j} \lambda, m\right)\right)^{-1} .
$$

Proof Since $\varphi \in \mathcal{S}(\mathbf{R} \times \mathbf{N})$, it is easy to prove

$$
\left(\Lambda_{1}+2\left(\Lambda_{2}+\frac{\partial}{\partial \lambda}\right)\right)^{j} m_{i}(\lambda, m) \leq C_{j}\left(4\left(m+\frac{\alpha+1}{2}\right)|\lambda|\right)^{-j}, \quad j=1,2, \ldots
$$

Then, Lemma 5 follows from Lemma 4.

By Lemma 5, we can prove

Theorem 3 If $s \in \mathbf{R}$ and $1<p<\infty$, we have

$$
\|f\|_{W_{p}^{s}} \sim\left\|\left(\sum_{j=0}^{\infty}\left|2^{j s} f * \varphi_{j}\right|^{2}\right)^{\frac{1}{2}}\right\|_{\alpha, p}, \quad f \in W_{p}^{s}(\mathbf{K}) .
$$


Proof For $f \in W_{p}^{s}(\mathbf{K})$, there exists $g \in L_{\alpha}^{p}(\mathbf{K})$ such that $f=J^{s} g$. Therefore,

$$
\begin{aligned}
\left\|\left(\sum_{j=0}^{\infty} r_{j}(t) 2^{j s} f * \varphi_{j}\right)\right\|_{\alpha, p} & =\left\|\left(\sum_{j=0}^{\infty} r_{j}(t) 2^{j s} J^{s} g * \varphi_{j}\right)\right\|_{\alpha, p} \\
& =\left\|g *\left(\sum_{j=0}^{\infty} r_{j}(t) 2^{j s} J^{s} \varphi_{j}\right)\right\|_{\alpha, p} .
\end{aligned}
$$

By Lemma 5 ,

$$
\left\|\left(\sum_{j=0}^{\infty} r_{j}(t) 2^{j s} f * \varphi_{j}\right)\right\|_{\alpha, p} \leq A_{1}\|g\|_{\alpha, p}=A_{1}\|f\|_{W_{p}^{s}} .
$$

Then

$$
\int_{0}^{1}\left\|\left(\sum_{j=0}^{\infty} r_{j}(t) 2^{j s} f * \varphi_{j}\right)\right\|_{\alpha, p} d t \leq A_{1}\|f\|_{W_{p}^{s} .}
$$

Following from the inequality (44) in [10],

$$
\begin{aligned}
\left\|\left(\sum_{j=0}^{\infty}\left|2^{j s} f * \varphi_{j}\right|^{2}\right)^{\frac{1}{2}}\right\|_{\alpha, p} & \leq C\left\|\int_{0}^{1}\left|\sum_{j=0}^{\infty} r_{j}(t) 2^{j s} f * \varphi_{j}\right| d t\right\|_{\alpha, p} \\
& \leq C \int_{0}^{1}\left\|\sum_{j=0}^{\infty} r_{j}(t) 2^{j s} f * \varphi_{j}\right\|_{\alpha, p} d t .
\end{aligned}
$$

Thus

$$
\left\|\left(\sum_{j=0}^{\infty}\left|2^{j s} f * \varphi_{j}\right|^{2}\right)^{\frac{1}{2}}\right\|_{\alpha, p} \leq C\|f\|_{W_{p}^{s} .}
$$

For the reverse, let $f=J^{s} g, k=\mathcal{F}^{-1}\left(\sum_{j=0}^{\infty} \varphi\left(2^{-2 j} \lambda, m\right)^{2} \hat{g}\right)$, by Lemma 5 again,

$$
\begin{aligned}
\|g\|_{\alpha, p} & =\left\|\mathcal{F}^{-1}\left\{\left(\sum_{j=0}^{\infty} \varphi\left(2^{-2 j} \lambda, m\right)^{2}\right)^{-1}\left(\sum_{j=0}^{\infty} \varphi\left(2^{-2 j} \lambda, m\right)^{2}\right) \hat{g}(\lambda, m)\right\}\right\|_{\alpha, p} \\
& \leq A_{2}\left\|\mathcal{F}^{-1}\left(\sum_{j=0}^{\infty} \varphi\left(2^{-2 j} \lambda, m\right)^{2} \hat{g}(\lambda, m)\right)\right\|_{\alpha, p}=A_{2}\|k\|_{\alpha, p} .
\end{aligned}
$$

Since

$$
\|k\|_{\alpha, p}=\sup _{\|\mu\|_{\alpha, q} \leq 1} \int_{\mathbb{K}} k(x, t) \mu(x, t) d m_{\alpha}(x, t)
$$

where $\frac{1}{p}+\frac{1}{q}=1$, we can choose $\mu \in L_{\alpha}^{q}(\mathbf{K})$ such that $\|\mu\|_{\alpha, q}=1$ and

$$
\int_{\mathbf{K}} k(x, t) \mu(x, t) d m_{\alpha}(x, t) \geq \frac{1}{2}\|k\|_{\alpha, p} .
$$


Let $w(x, t)=J^{-s} \mu(x, t) \in W_{q}^{-s}(\mathbf{K})$. Then

$$
\left.\hat{f}(\lambda, m) \hat{w}(\lambda, m)=\widehat{\left(J^{s} g\right.}\right)(\lambda, m) \widehat{\left(J^{-s} \mu\right)}(\lambda, m)=\hat{g}(\lambda, m) \hat{\mu}(\lambda, m) .
$$

Therefore,

$$
\begin{aligned}
\|f\|_{W_{p}^{s}}= & \|g\|_{\alpha, p} \leq C \int_{\mathbf{K}} k(x, t) \mu(x, t) d m_{\alpha}(x, t) \\
= & C \int_{\mathbf{R} \times \mathbf{N}} \hat{k}(\lambda, m) \hat{\mu}(\lambda, m) d \gamma_{\alpha}(\lambda, m) \\
= & C \int_{\mathbf{R} \times \mathbf{N}} \hat{\mu}(\lambda, m)\left(\sum_{j=0}^{\infty} \varphi\left(2^{-2 j} \lambda, m\right)^{2} \hat{g}(\lambda, m)\right) d \gamma_{\alpha}(\lambda, m) \\
= & C \int_{\mathbf{R} \times \mathbf{N}} \sum_{j=0}^{\infty}\left\{\left(2^{j s} \hat{f}(\lambda, m) \varphi\left(2^{-2 j} \lambda, m\right)\right)\right. \\
& \left.\times\left(2^{-j s} \hat{w}(\lambda, m) \varphi\left(2^{-2 j} \lambda, m\right)\right)\right\} d \gamma_{\alpha}(\lambda, m) \\
= & C \int_{\mathbf{K}} \sum_{j=0}^{\infty}\left\{\left(2^{j s}\left(f * \varphi_{j}\right)(x, t)\right)\left(2^{-j s}\left(w * \varphi_{j}\right)(x, t)\right)\right\} d m_{\alpha}(x, t) \\
\leq & C\left\|\left(\sum_{j=0}^{\infty} 2^{j s}\left|f * \varphi_{j}\right|^{2}\right)^{\frac{1}{2}}\right\|\left\|_{\alpha, p}\right\|\left(\sum_{j=0}^{\infty} 2^{-j s}\left|w * \varphi_{j}\right|^{2}\right)^{\frac{1}{2}} \|_{\alpha, q} .
\end{aligned}
$$

By (8),

$$
\left\|\left(\sum_{j=0}^{\infty} 2^{-j s}\left|w * \varphi_{j}\right|^{2}\right)^{\frac{1}{2}}\right\|_{\alpha, q} \leq C_{2}\|w\|_{W_{q}^{-s}}=C_{2}\|\mu\|_{\alpha, q}=C_{2} .
$$

Thus

$$
\|f\|_{W_{p}^{s}} \leq C\left\|\left(\sum_{j=0}^{\infty} 2^{j s}\left|f * \varphi_{j}\right|^{2}\right)^{\frac{1}{2}}\right\|_{\alpha, p} .
$$

Then Theorem 3 follows from (8) and (9).

The following lemma has been proved in [12].

Lemma 6 Let $1 \leq P_{0}, P_{1}<\infty, 0<\theta<1$ and $\frac{1}{p}=\frac{1-\theta}{p_{0}}+\frac{\theta}{p_{1}}$.

(1) If $\left\{A_{0}, A_{1}\right\}$ is an interpolation couple, then

$$
\left[L_{p_{0}}\left(A_{0}\right), L_{p_{1}}\left(A_{1}\right)\right]_{\theta}=L_{p}\left(\left[A_{0}, A_{1}\right]_{\theta}\right) .
$$

(2) If $A_{j}, j=1,2, \ldots$ are Banach spaces and

$$
l_{p}\left(A_{j}\right)=\left\{a: a=\left\{a_{j}\right\}_{j=1}^{\infty}, a_{j} \in A_{j},\|a\|_{l_{p}\left(A_{j}\right)}=\left(\sum_{j=0}^{\infty}\left\|a_{j}\right\|_{A_{j}}^{p}\right)^{\frac{1}{p}}<\infty\right\},
$$


where $1 \leq p<\infty$ and $\left\{A_{j}, B_{j}\right\}, j=1,2, \ldots$ are interpolation couples, then we have

$$
\left[l_{p_{0}}\left(A_{j}\right), l_{p_{1}}\left(B_{j}\right)\right]_{\theta}=l_{p}\left(\left[A_{j}, B_{j}\right]_{\theta}\right) .
$$

Now we can prove the main result of this section.

Theorem 4 Let $1<p, q<\infty$ and $s \in \mathbf{R}$. Then

$$
F_{p, q}^{S}(\mathbf{K})=\left[W_{p_{0}}^{s_{0}}(\mathbf{K}), B_{p_{1}, p_{1}}^{s_{1}}(\mathbf{K})\right]_{\theta},
$$

where $s_{0}, s_{1} \in \mathbf{R}, 1<p_{0}, p_{1}<\infty, 0<\theta<1, s=(1-\theta) s_{0}+\theta s_{1}$, and $\frac{1}{p}=\frac{1-\theta}{p_{0}}+\frac{\theta}{p_{1}}, \frac{1}{q}=\frac{1-\theta}{2}+\frac{\theta}{p_{1}}$.

Proof By Theorem 1 and Theorem 3, it is sufficient to prove

$$
\left[\left\|\left(\sum_{j=0}^{\infty}\left|2^{j s_{0}} f * \varphi_{j}\right|^{2}\right)^{\frac{1}{2}}\right\|_{\alpha, p_{0}},\left\|\left(\sum_{j=0}^{\infty}\left|2^{j s_{1}} f * \varphi_{j}\right|^{p_{1}}\right)^{\frac{1}{p_{1}}}\right\|_{\alpha, p_{1}}\right]_{\theta}=\left\|\left(\sum_{j=0}^{\infty}\left|2^{j s} f * \varphi_{j}\right|^{q}\right)^{\frac{1}{q}}\right\|_{\alpha, p} .
$$

Let $A_{j}=2^{j s_{0}} \mathbb{C}, B_{j}=2^{j s_{1}} \mathbb{C}$, where $\mathbb{C}$ is the set of complex numbers. Then, by Lemma 6 , we can get our theorem $(c f .[12])$.

By the properties of the Sobolev space and the Besov space, we can get the following properties of the Triebel-Lizorkin space on $\mathbf{K}$.

\section{Proposition 7}

(1) Let $1<p<\infty, 1<q_{0}<q_{1}<\infty, s \in \mathbf{R}$. Then

$$
F_{p, q_{0}}^{s}(\mathbf{K}) \subseteq F_{p, q_{1}}^{s}(\mathbf{K})
$$

(2) Let $1<p<\infty, 1<q_{0}<q_{1}<\infty, \epsilon>0$. Then $F_{p, q_{0}}^{s+\epsilon}(\mathbf{K}) \subseteq F_{p, q_{1}}^{s}(\mathbf{K})$.

(3) Let $1<p<\infty, 1<q<\infty, s \in \mathbf{R}$. Then

$$
B_{p, \min \{p, q\}}^{s}(\mathbf{K}) \subseteq F_{p, q}^{s}(\mathbf{K}) \subseteq B_{p, \max \{p, q\}}^{s}(\mathbf{K})
$$

(4) $W_{p}^{s}(\mathbf{K})=F_{p, 2}^{s}(\mathbf{K}), 1<p<\infty, s \in \mathbf{R}$.

(5) $\left(F_{p, q}^{s}(\mathbf{K})\right)^{\prime}=F_{p^{\prime}, q^{\prime}}^{-s}(\mathbf{K})$, where $1<p, q<\infty, s \in \mathbf{R}$ and $\frac{1}{p}+\frac{1}{p^{\prime}}=1, \frac{1}{q}+\frac{1}{q^{\prime}}=1$. 


\section{References}

1. Assal, M, Abdallah, HB: Generalized Besov type spaces on the Laguerre hypergroup. Ann. Math. Blaise Pascal 12 , 117-145 (2005)

2. Assal, M, Nessibi, MM: Sobolev spaces on the dual of the Laguerre hypergroup. Potential Anal. 20, 85-103 (2004)

3. Bergh, J, Löfström, J: Interpolation Spaces: An Introduction. Springer, Berlin (1976)

4. Bloom, WR, Heyer, H: Harmonic Analysis of Probability Measures on Hypergroups. de Gruyter Studies in Mathematics, vol. 20. de Gruyter, Berlin (1994)

5. Cruz-Báez, DI, Rodríguez, J: Spaces of distributions of Besov and Triebel-Lizorkin type for the Fourier-Bessel transform. J. Math. Anal. Appl. 259, 51-63 (2001)

6. Folland, G, Stein, EM: Hardy Spaces on Homogeneous Groups. Princeton University Press and University of Tokyo Press, Princeton (1982)

7. Huang, JZ, Liu, HP: Weak $(1,1)$ type estimates of the maximal functions on the Laguerre hypergroup. Can. Math. Bull. $53,491-502(2010)$

8. Huang, JZ: Littlewood-Paley $g$-functions and multipliers for the Laguerre hypergroup. J. Inequal. Appl. 2011, ID $741095(2011)$

9. Jewett, Rl: Spaces with an abstract convolution of measures. Adv. Math. 18, 1-101 (1975)

10. Stein, EM: Singular Integrals and Differentiability Properties of Functions. Princeton University Press, Princeton (1970)

11. Stempak, K: Mean summability methods for Laguerre series. Trans. Am. Math. Soc. 322, 671-690 (1990)

12. Triebel, H: Interpolation Theory, Function Spaces, Differential Operators. North-Holland, Amsterdam (1978)

doi:10.1186/1029-242X-2012-190

Cite this article as: Huang: Sobolev space, Besov space and Triebel-Lizorkin space on the Laguerre hypergroup. Journal of Inequalities and Applications 2012 2012:190.

\section{Submit your manuscript to a SpringerOpen ${ }^{\circ}$ journal and benefit from:}

- Convenient online submission

- Rigorous peer review

- Immediate publication on acceptance

Open access: articles freely available online

- High visibility within the field

- Retaining the copyright to your article 